\title{
Christian Prigent : la distance et l'émotion
}

\author{
Roger-Michel Allemand \\ Laboratoire Babel - Université du Sud Toulon - Var
}

Roger-Michel Allemand - Commençons par le biais d'un de nos amis communs, Denis Roche, sur l'œuvre duquel vous avez écrit votre premier essai (Prigent, 1969a). Il m'a souvent dit son «impression d'être sur une arête sagittale d'intrusion dans la littérature » (Roche et Allemand, 2001, p.298). Qu'en est-il pour vous?

Christian Prigent - Je ne dirais plus désormais que ceci : primo, mes textes sont exactement ce que, sans pourtant savoir a priori ce qu'ils seraient, j'ai voulu qu'ils fussent; deuzio, personne d'autre n'a écrit cela, comme cela. Ces écrits font consister la langue que j'ai cherchée : celle qui restitue le mieux possible l'effet que le monde me fait. Y compris dans leurs 
maladresses et leurs palinodies. Tant mieux si leur singularité stylistique, l'écart que dessine cette singularité et la pensée qu'appelle théoriquement cet écart posent quelques questions à ceux qui s'intéressent encore à ce que vous et moi appelons « littérature ». Ce que disent ceux qui commentent ici ou là mes livres va parfois dans ce sens. Mais il ne me reste pas grandchose de l'enthousiasme naïf qu'il faut pour se vouloir à la pointe des opérations de réinvention artistique. Il faut d'ailleurs se faire à ce constat : la littérature n'intéresse pas grand monde. De moins en moins de monde, même. Ça peut inciter ceux qui s'y adonnent à une certaine modestie quant à l'effet des « intrusions » qu'ils pourraient y opérer.

R.-M. A. - Certes, mais vous parlez de « geste d'effraction dans le corps de la langue. Elle cherche à y faire craquer les coutures épidermico-sémantiques. » (Prigent et Gorrillot, 2009, p. 67). Il y a donc de la violence dans votre poésie. Jusqu'à quel point en est-elle constitutive?

C. P. - Je suis parti, il y aura bientôt cinquante ans, du constat que les formes écrites de représentation qui étaient à disposition ne faisaient pas résonner en moi la note juste de l'expérience que j'avais du monde (le spectaculaire dehors comme l'intime dedans). Y compris les formes poétiques qui me séduisaient pourtant violemment. Il m'a fallu aller voir comment ça marchait dans ces dernières : Rimbaud ${ }^{1}$, Ponge $^{2}$, Denis Roche ${ }^{3}$... Aller voir dedans, c'était certes rendre à ces œuvres l'hommage de l'admiration. Mais c'était aussi travailler

\footnotetext{
1 Voir Prigent et Gorrillot, p. [29-31].

2 Voir Prigent, 1971, 1972b, 1972c, 1977c, 1978b, 1989b, 1999b.

3 Voir Prigent, 1974a, 1974b, 1974c, 1976, 1977a, par exemple, et pour l'intérêt réciproque, cf. Roche, 1977, notamment.
} 
à détraquer (par l'analyse, le pastiche et la parodie) les mécaniques qui y fonctionnaient. Ensuite il fallait remettre en service des petits circuits " poétiques » avec tous ces ressorts et rouages désœuvrés puis autrement remontés et réactivés. C'est une "violence», oui, si on veut. Et elle est "constitutive», certes. Mais il faut bien dire aussi que ce n'est guère davantage qu'une opération de recyclage, un bricolage empirique et tâtonnant.

R.-M. A. - Mais qu'est-ce qui a été blessé dans la langue ? Et d'ailleurs, quelle langue? Le «corps de prescriptions et d'habitudes » (Barthes, 1972, p. 11) ?

C. P. - Il ne s'agit pas de la langue en soi (du corpus verbal et de ses formes artistiquement stylisées). Il s'agit du réseau des représentations que tresse l'usage contractuel de la langue et qu'il impose comme lieu idéologique commun. Il s'agit en somme de la « réalité » : du monde identifié à la somme des représentations qu'on en a. Et de la puissance d'aliénation qu'est l'assignation des sujets à ce réseau de représentations contraignantes. La littérature et la poésie académiques (y compris l'académisme moderniste) sont des composantes du réseau, au même titre que la vulgate des discours (science, morale, politique...) qui au jour le jour médiatisent pour nous le monde. Qui écrit rêve de s'extirper du réseau que je dis et de se soulager un peu du poids de l'aliénation. Et donc tente des opérations sur le réseau : recompositions défigurées des figures qu'il dessine, redécoupages, distorsions parodiques, précipitations rythmées, pulvérisations sonorisées. Ce n'est pas pour «blesser ». Mais pour faire de l'air. Pour raviver la langue (moribonde de n'être que dénominateur commun et vecteur 
d'assentiment soumis). Et pour essayer d'ouvrir de petits espaces de sensation fraîche et de sens vivant.

R.-M. A. - «Une seule issue : parler contre les paroles », disait Ponge (1965, p. 186). Se couper de la poésie subjective, soit; mais puisque l'espace poétique est devenu voyou, pour paraphraser l'une des formules que vous utilisez (Prigent, 1977b, p.126), à quelles intentions répond désormais l'invention?

C. P. - La phrase «l'espace est resté voyou et il est difficile d'énumérer ce qu'il engendre » provient d'un bref article de Documents ${ }^{4}$. Elle est d'un Georges Bataille extasié par le défi qu'opposent l'engendrement et la dépense de l'univers « acéphale » à l'effort de symbolisation poétique. Si l'invention a un but, c'est de relever ce défi : former des formes informées par cette pression informe. Dit ainsi, c'est d'une prétention un peu exorbitante (le souvenir du ton "grand seigneur» de Bataille porte à cette emphase). On saisira mieux ce que je veux dire si on mesure l'écart (chacun de nous en fait banalement l'épreuve) entre la mesure grammaticale et rhétorique et la complexité stricto sensu dé-mesurée de l'expérience individuée. L'exigence d'invention naît de ce constat. Et tente envers et contre tout de former en langue quelque chose de la complexité dont je parle.

R.-M. A. - Et à quelles aspirations répond l'impulsion créatrice?

C. P. - À ce que je viens de dire, je crois : le projet de trouver une langue qui réponde le mieux possible aux défis qui sont à la source du besoin d'écrire; la résolution de ne rien

${ }^{4}$ Voir Bataille, 1930. 
céder sur le désir que cette langue soit, sans concession au bruitage d'époque, sans peur des tentatives d'intimidations scolaires ou mondaines; la certitude que rien du monde ne se perçoit ni ne se comprend en vérité si cette langue-là ne vient pas médiatiser l'expérience d'une façon plus intimement juste qu'aucune autre des médiations (intellectuelles, savantes, religieuses, idéologiques...) que propose l'époque ; la volonté de jouir des effets de «nouveau » qui se dégagent de la trouvaille de cette langue; l'espoir, enfin, que tout cela puisse communiquer à quelques-uns, dits «lecteurs», un peu de la liberté et de la jubilation qu'en se faisant ça s'est octroyé.

R.-M. A. - Le titre Power/powder apparaît comme un contrepied au flower power attribué à Ginsberg. N'y a-t-il pas cependant des points communs entre votre démarche d'alors et celle de la beat generation? Un rejet du lyrisme bucolique, du conformisme moral et esthétique, au profit d'un autre lyrisme?

C. P. - Le titre auquel vous vous référez voulait finement (?) introduire le coup du « $\mathrm{d}$ » entre des blocs de langue de pouvoir (des énoncés politiques et publicitaires) et la poudre en laquelle le geste d'écriture était censé les réduire. J'ai écrit ce livre en 1973-1974. Pas de référence consciente au "flower power », non. Mais un volontarisme maoïsto-carnavalesque un peu trop raide de la nuque programmative pour pouvoir accoucher d'autre chose que d'une crispation kitsch des marottes d'époque. Les poètes beat (surtout Ginsberg et Corso) avaient joué un rôle déterminant pour moi. Mais bien avant Power/powder. Je les avais découverts dans l'anthologie publiée en 1965 chez Denoël par Jean-Jacques Lebel. Peut-être ne mesure-t-on pas assez aujourd'hui l'effet d'irruption de ces textes dans le mièvre jardinage poétique à la française et les 
ultimes bricolages des surréalistes. Un autre lyrisme, incroyablement exalté et violent: emportement rythmique, oralité implicite, lexique non a priori « poétique», décor urbain moderne, rébellion politique, obscénité radieuse, etc. J'ai écrit directement sous cette influence pendant pas mal de mois, en 1966-1967.

R.-M. A. - Cela étant, la beat generation s'inscrivait dans le nomadisme - géographique, social et intellectuel (pas seulement Kerouac, Robinson Jeffers aussi, par exemple) alors que vous me semblez davantage ancré, si je puis dire, à la Bretagne, à vos origines familiales, à tout votre patrimoine culturel. Est-ce à votre avis un écart structurant?

C. P. - Sans doute, oui. Mais de toute façon je me suis vite éloigné du lyrisme un peu oratoire de la poésie beat. Le néobouddhisme de Ginsberg et ses duos folk avec Orlovsky à la guitare, ça me laissait très... froid. Et l'errance érémitique, la "route», les paradis artificiels et le mysticisme new age à Big Sur, non, très peu pour moi. Mais je me suis ensuite, dans les années 1970-1980, beaucoup intéressé au Burroughs de Soft machine et du Job. Pour le mythe opératoire que ces textes construisent : la langue pensée comme un virus introduit dans le corps animal. Et surtout pour la mise en œuvre des techniques de cut-up et la portée théorique (la «morale») de cette méthode de travail. J'en ai tiré quelques leçons pour forger mes propres outils - ceux dont je me sers surtout dans les proses publiées chez P.O.L depuis Commencement. Des livres qui ont effectivement traité un matériau de plus en plus référé au monde géographique, idéologique et culturel de mon enfance (la Bretagne, les communistes, etc.). 
R.-M. A. - La recherche des marges - au double sens de la dissidence et de la mise en page - n'est-elle pas un fil conducteur de votre travail ?

C. P. - Chercher «sa » langue voue aux marges, c'est inéluctable: il faut donner forme verbale à une dissidence, refuser le «lieu commun»des représentations (éprouvées comme inappropriées, défectueuses). Après ce geste inaugural de défiance (qu'avive la fréquentation de ceux qui le firent avant vous - pour moi ce fut essentiellement Rimbaud), on travaille à noter ce qui apparaît de surprenant, de frais (ça veut dire au fond: de juste et de beau) dans les marges différentielles qu'on s'est ménagées à côté des propositions consensuelles de l'imaginaire d'époque. Ça n'apparaît évidemment pas à volonté. Ça naît dans les espaces (des bords, des suspens, des failles, des accélérations ou des ralentis rythmiques) qu'on ouvre par le travail formel, les ruses de l'élaboration rhétorique, les petites opérations stratégiques qui visent à laisser la langue jouer ses propres aventures. Dit ainsi, je vois bien que c'est un peu vague. Pour me faire mieux comprendre: l'abandon aux échos homophoniques (dont la rime elle-même), la composition par montage de prélèvements cut-upées, la dictée des contraintes métriques, etc., relèvent de ces opérations négatives d'ouverture (une narrativité ou une expressivité que ne domine pas un vouloir-dire a priori et où les effets d'énonciation débordent toujours les segments énoncés).

R.-M. A. - Exil pas si facile donc. «La solitude est ce à quoi voue la puissance d'ex-ception de l'écriture (ou, en termes mallarméens, son effet de retranchement). " (Prigent et Gorrillot, 2009, p. [31]), écrivez-vous à propos de Beckett. De l'œuvre comme une forteresse donc? 
C. P. - Socialement parlant, une certaine solitude intellectuelle, affective et physique est la conséquence de ce que je viens de dire. Elle réalise les effets de cette singularité qui poussa inauguralement à chercher, par l'écriture, la symbolisation d'une différence et à faire de cette symbolisation l'instrument d'une connaissance plus juste de soi et du monde. Ce n'est pas abstrait : j'ai vécu cela toute ma vie. En clair, ça veut dire qu'il faut vivre généralement loin des quelques-uns avec qui il y aurait connivence, fraternité, loyauté, lieu (éthique, esthétique et intellectuel) largement commun; et côtoyer au jour le jour les autres, inéluctablement étrangers, presque toujours indifférents, souvent innocemment menaçants, parfois effectivement agressifs. Une sorte d'exil sur place, en somme. Mais il n'y a pas de quoi en faire un plat héroïsé. D’abord parce qu'on est la plupart du temps occupé à autre chose qu'écrire et donc logé au lieu le plus misérablement commun. Ensuite parce qu'on ne se vit pas comme un Achille gonflé de la conscience de sa propre exception, replié dans sa «forteresse » obsidionale et toisant de haut l'ingratitude du monde. C'est tout le contraire : l'œuvre, toute hérissée d'opacités et d'étrangetés qu'elle soit (voire du fait même de ces bizarreries) se veut intensément espace et temps de communication. Une communication certes paradoxale, puisque ne tâchant à communiquer que ce qui renâcle à l'immédiateté transparente de la "communication » courante. Activant un autre régime de la communication: communication d'une expérience fondée sur ce qui, ne se communiquant pas dans la langue du contrat social «commun », frappe de soupçon la notion même de communication (l'échange des savoirs utiles, des mirages consommateurs et des affects stéréotypés). Mais communication quand même, parce que, dans un espace certes tramé par la désillusion et 
rétif à toute effusion conviviale, l'œuvre offre de partager des blocs d'expérience à la fois savante et sauvage, sensoriellement fixés en langue (Bataille appelait ça du «non savoir ») — et d'en tirer une chance d'échapper aux assignations qui nous vouent justement à n'être que des sujets aliénés au bas régime de la communication hyper-médiatique.

R.-M. A. - Mais comment concilier la crise rimbaldienne et la prise éminemment politique de votre écriture sur le monde?

C. P. - Ce que je viens d'essayer de dire attribue au travail de littérature une fonction d'émancipation. C'est en ce sens que la crise qui fait qu'il y a de l'écriture (plutôt que seulement la gestion pragmatique, mondaine ou savante des discours positifs) a une signification de part en part politique. Étant entendu que cette cause (ce qui fait écrire) et cet effet (ce que fait écrire) ne sont d'évidence «politiques» qu'au lieu auquel atteignent (et attentent) les gestes poétiques : le lieu des représentations, le lieu des noms et des figures, le lieu où se constitue le réseau verbalisé de l'idéologie. Et non pas, bien sûr, directement, le lieu de l'action pratique et objectivement transformatrice. Aucune compréhension de la prise politique d'une écriture n'est possible si on ne part pas de cette évidence. Et, si on n'en tire pas les conséquences, on ne peut que s'enferrer dans des impasses. C'est-à-dire nier la spécificité de la littérature, rabattre le régime spécial de "communication » dont nous parlions à l'instant sur le modèle banal de la communication et livrer la souveraineté poétique à des sommations naïves : «s'engager», « déclarer» le vrai, parler «au nom de», «agir» immédiatement et efficacement. Ces sommations ne sont jamais que des tentatives d'intimidation. 
Elles ne tiennent que d'une ignorance (toujours intéressée) des modes particuliers d'exercice du travail poétique. Au bout du compte, elles sont la négation de ses raisons d'être et d'inventer des formes adéquates aux enjeux d'époque. De mon propre travail (qui n'a cessé, vous le savez bien, de se poser ce type de question) et de son éventuelle "prise politique sur le monde» je ne saurais dire rien de plus que cela. J'ai écrit un jour : «la poésie peut peu». Je le répète. J'ai écrit aussi que je ne me passerai jamais de la conviction que la même poésie a une « responsabilité civique ». Je répète cela aussi. Sur ce qu'il en est plus en détail de cet impouvoir, de cette responsabilité et de leur articulation problématique, je ne peux que renvoyer à tout ce que j'ai déjà dit dans plusieurs livres et dont j'assume, parce qu'en l'occurrence rien ne peut être simple, toutes les propositions, voire les approximations, les palinodies et même les contradictions.

R.-M. A. - L'avant-garde est un renversement des élites et du pouvoir établi, mais au-delà du principe d'avant-garde, comme vous l'écriviez en 1981, n'est-ce pas aussi que votre œuvre entend créer un autre monde, qui se substitue à la réalité commune?

C. P. - Il n'y a pas d'autre monde. Par contre il y a le monde comme autre, comme altérité à ce que j'appelais tout à l'heure "réalité» - et qui est une fiction que les représentations dominantes cherchent à nous faire prendre pour le réel ${ }^{5}$. Ce que j'écris essaie de faire monter dans des formes adéquates des traces de cette altérité. Ce qui ne veut pas dire la figurer ou la nommer : elle relève en son principe d'un

5 Voir Napoli, 2004. 
infigurable et d'un innommable. Mais en révéler, dans la balistique de l'écrit (rythmes, traces d'énonciation, liaisons sémantiques injustifiables), l'écart génératif ; poser cet écart comme la condition nécessaire et suffisante pour qu'il y ait de l'écriture ; et parier que de cette instance négative peut naître non pas un autre monde formé (représenté), mais une telle puissance de déformation des mondes habitués qu'aucune fiction stable ne puisse y coaguler et venir, sous le nom de « réalité », faire écran à la vérité de l'expérience singulière.

R.-M. A. - Quel a été le rôle de la théorie littéraire sur vos propres pratiques?

C. P. - Un rôle décisif au moment où il m'a fallu essayer de comprendre pourquoi ce que j'étais spontanément porté à écrire (vite dit: un lyrisme post-surréaliste et diverses variantes du vers-librisme standard façon années 1950-1960) me décevait radicalement: à la fois surexcité et insipide, impropre à penser l'impensé "moderne», sans prise sur ma propre souffrance et ma propre jouissance. Puis un rôle très important, en alternance systématique avec le travail de fiction, tout au long de mon parcours. Aucune contradiction, pour moi, entre ces deux postures intellectuelles. $\mathrm{Ni}$, je crois, de perte de l'une dans l'autre, de développement de l'une aux dépens de l'autre. Parce que je pense, comme Sade l'écrit dans Justine, que c'est «en n'analysant jamais rien qu'on s'aveugle sur tout et qu'on se prive de toutes les jouissances " ${ }^{6}$. Et, symétriquement, qu'on n'accède à aucune pertinence analytique, à aucune envergure théorique, à aucune connaissance profonde si on ne sait pas, en pratique, se laisser déborder par ces au-delà (ou endeçà ?) de l'utilitarisme rationnel et de l'homogénéité

${ }^{6}$ Voir Prigent, 1978a en particulier. 
conceptuelle que sont les dérives poétiques dans le jeu ignorant et la violence hétérogène de l'expérience.

R.-M. A. - Vos textes sont techniquement très maîtrisés, vos livres de poèmes ne sont pas de simples recueils, mais sont construits en tant qu'ouvrages. C'est un peu paradoxal, non, ce sens aigu de l'ordre pour un écrivain qui veut libérer toute la potentialité des mots?

C. P. - Spontanément, on n'exprime que des croyances reçues, des fadaises psychologiques, des représentationsclichés et des fantasmagories surcodées. Forcément : c'est cela qui nous permet de tenir face au chaos du monde ; et c'est donc cela que les filtres symboliques nous laissent imaginer et nous autorisent à dire. On ne sort pas de ça par les moyens qui font que ça est: ceux de l'expressivité réflexe. Les réussites de l'écriture automatique façon surréaliste sont dans quelques poèmes où une structure ostensiblement ordonnée appelle et cadre impérativement le jeu des associations libres (le plus bel exemple est pour moi «Union libre» d'André Breton). Sinon : défilé de souvenirs non analysés, "merveilleux» convenu et idéalisé, bric-à-brac onirique déballé dans une syntaxe intouchée. Guère d'intérêt. On retient de l'expérience des débuts (au temps des Champs magnétiques) une volonté de faire dégorger de la langue autre chose que les stéréotypes de l'expression lyrique. J'aime la générosité, la joie et le courage de cette réponse à l'appel de l'altérité. Mais moi, j'essaie plutôt d'assécher l'expressionnisme, de calmer les impulsions du vouloir-dire, d'échapper aux chromos stylés et aux idées reçues. Pour cela: artefact délibéré, tactiques de résistance, stratégie de disposition des obstacles rhétoriques, composition de pièges tendus à la spontanéité, effort vers des formes à la fois denses 
et fluides. En somme: multiplication des manipulations (formelles, rhétoriques) pour ménager les chances d'apparition d'un sens "inouï». L'inouï, c'est l'irruption du sensible-vrai formalisé au travers de l'expression réflexe et contre ses distractions. Une excursion hors du pensé habitué et de la modulation stylée des représentations : une ex-tase, en somme, si on débarrasse ce terme d'un peu d'emphase et de beaucoup d'implications inutilement mystiques. Le sens de l'opération (son axe, son mouvement, sa composition dynamique de l'intelligible) réside dans l'invention de formes où l'afflux des figures, des images, des significations, à la fois fait système et prend en masse, à la fois se laisse déborder, défaille et s'évanouit rythmiquement dans une motilité qui voue ces formes elles-mêmes à un excès définitivement instable.

R.-M. A. - Car tout part des mots, n'est-ce pas ?

C. P. - Nous parlons ici de littérature. La littérature se fait avec des mots. Tout part donc de là. Et y revient. Sauf que dans ce parcours, quelque chose d'autre est impliqué : quelque chose qui vient comme altérité, justement. Dont il y a peu à dire, sinon ceci : c'est une altérité ; elle est non logique; elle est ce que vise (à symboliser) l'opération d'écriture; et c'est parce qu'il y a cette intuition de l'altérité que l'effort d'écriture rumine son ex-cès et mijote son ex-tase. Dit autrement : l'organisation symbolique comme structuration et expansion du nommable sécrète l'intuition qu'il y a de l'innommable et assigne à la littérature la tâche non pas de nommer cet innommable mais de maintenir dans la clôture du nommé une ouverture innommable, un flottement, un jeu - qui est une condition de vérité et une chance de désaliénation. Tout cela découle de l'inconfortable statut du parlant : soumis d'une part à l'exigence 
incontournable de représenter sans cesse sa vie (empêché, du fait qu'il parle, de seulement la vivre) ; découvrant d'autre part l'impossibilité de ladite représentation (son impertinence foncière, en tout cas sa désastreuse approximation); et s'agitant séculairement, face aux effets énervants de ce double bind, pour les compenser par l'abrutissement simple, l'exaltation illuminée ou la dévotion aux savoirs positifs.

R.-M. A. - J'évoquais en ouverture de nos échanges votre essai de 1969 : il y a bien un avant et un après. C'est d'ailleurs cette année-là que vous fondez TXT. Auparavant, votre inspiration a quelque chose à voir avec une espèce de flux et d'enthousiasme. Ensuite, le phrasé du geste poétique s'épure. L'ôteur de Power/powder, il ôte quoi?

C. P. - Je vais me répéter: l'auteur de Power/powder sortait de quatre ou cinq ans de règlements de comptes théoriques et pratiques avec celui qu'il avait poétiquement été : un post-surréaliste idéaliste et torrentiel (un peu remusclé et modernisé quand même par la fréquentation des poètes beatniks). Il avait fait chez Ponge sa cure anti-lyrique d'objectivité «matérialiste» et rectifié un peu sa volubilité expressionniste au contact de la "mécriture » iconoclaste de Denis Roche ${ }^{7}$. Il découvrait qu'on n'écrit pas à partir d'un contenu hors langue limité, homogène et stable (qu'il y aurait à décrire, raconter ou exprimer par des moyens verbaux adéquats). Mais qu'on œuvre dans et contre la « réalité », qui est toujours-déjà une Darstellung, un imaginaire au sens kantien en tout cas une composition de langue proposée comme «monde». Il voulait que l'effort au style approche un peu de l'expérience et aille, au travers des significations déjà

7 Voir Roche, 1972. 
constituées, vers quelque chose d'innommable, voire d'extatiquement in-signifiant (dit alors : «réel »). Pour ce faire, il fallait quitter la posture auctoriale: ne pas augmenter mais ôter au donné naturalisé des représentations d'époque; et travailler à trouer l'écran dit "réalité », à en défaire la cohérence, à en distendre le maillage - à le priver des conditions d'exercice de sa domination péremptoire.

R.-M. A. - «Écrire ne signifie rien, n'a aucun sens, ne rassure nul à quoi bon, ne comble nul vide, n'équivaut à rien, n'a lieu qu'à excéder tout sens et toute équivalence (toute réclusion dans l'horizon du savoir et du discours). " (Prigent, 2005, p. 141) Mais quid de la plus-value de sens à la lecture : est-elle remplacée par des effets de sens - aux niveaux sémantique, syntaxique, prosodique, phonique?

C. P. - Le «sens" réside pour moi dans l'invention concertée de la forme. Il ne s'identifie en rien à la somme des significations que le texte distribue en segments narratifs (scènes, actions), descriptifs (paysages, figures) ou conceptuels (métatechniques, méditatifs). Il passe (je veux dire : il naît, il circule et il meurt) dans des moments où le réseau des significations intelligibles est à la fois tramé et aéré par d'autres instances de production (échos sonores, rebonds homophoniques, prosodie métrée, jeux intertextuels, composition fuguée...) qui y activent un phrasé cinématique. Peu importe en définitive le matériau que cet usinage traite (souvenirs, fantasmes, imageries d'enfance, démêlés avec la

\footnotetext{
8 Ce texte est un extrait de celui livré dans le collectif WOZU Dichter in dürftiger Zeit, Soleil noir éditeur, 1978 (le titre de l'ouvrage reprend l'interrogation de Hölderlin dans son élégie «Brot und Wein » : "Pourquoi des poètes en un temps de détresse?»). Voir Prigent, 1993.
} 
parentèle, aventures et mésaventures d'Éros, vignettes d'Histoire...). Je sais bien que, spontanément, c'est au matériau qu'une lecture s'intéresse d'abord. J'admets même que ce soit légitime. Mais c'est pourtant bien le complexe formel qui fait sens: il dit pourquoi c'est fait et développe les effets d'inventivité instable que ça veut produire. J'ajouterai que si on veut garder la complexité et la fraîcheur, il faut ré-usiner sans cesse, résister au maniérisme, maintenir quelque chose de l'énergie des commencements, rester un enfant : joueur, étonné, irrésolu, inaccompli, insaisissable - tout en étant aussi le contraire de cet enfant : rationnel, froid, cultivé, technique et tactique. Ce qui me convient, c'est de maintenir cette contradiction dans des séances d'écriture qui peu à peu font des livres. De l'effort de recommencement dont je parle témoigne par exemple le «roman en vers » qu'est Météo des plages : il est le résultat d'une volonté délibérée (et précisément programmée) de prendre le contre-pied de ce à quoi j'avais abouti avec Grand-mère Quéquette et surtout avec Demain je meurs (une prose rythmiquement emportée, l'oralité à fleur d'énonciation, une certaine lisibilité narrative, le contenu autobiographique quasi explicite, etc).

R.-M. A. - Sans vouloir être provocateur, qu'en est-il d'une éventuelle profondeur? Disant cela, je pense bien sûr à plusieurs de vos textes, mais aussi à votre ancien intérêt pour les peintres de Support/Surface (voir Prigent et Gorrillot, 2009, p. [151]).

C. P. - Si l'écrit n'est pas intérieurement inactif (si le détail du signifiant y joue ses minuscules aventures : rimes, métrique, jeux de mots, pistes sémantiques multiples...), il y a une sorte de creusement de la surface (le champ de propulsion 
axiale des significations). Le travail de phrasé dont je parlais à l'instant est la forme que prend, acoustiquement et visuellement, cette suggestion d'une profondeur ouverte dans ce qui est pourtant donné comme tout entier joué en surface. La forme que je cherche est complexe. Mais elle ne suppose caché aucun secret signifiant, aucun « mystère ». Il n'y a aucun recel de «profondeur», au sens d'une vérité ésotériquement dérobée. Tout est compris dans la surface du texte. Les « aventures du signifiant » ont un effet « creusant » parce qu'ils compliquent et épaississent la ligne des associations sémantiques. Mais ils sont seulement la condition d'activité du texte, ce qui fait qu'il y a en lui la trace d'une énonciation vivante, qu'il ne se réduit pas à la somme des énoncés qu'il aligne. La profondeur, c'est cette motilité. Et puisque vous évoquez Support/Surface : la profondeur, chez un Viallat, n'estelle pas dans le passage potentiellement infini de la forme biomorphiquement ambivalente qui creuse le support surfacial, pourtant affirmé comme tel, d'une puissance illimitée de suggestion sensuelle? Et n'est-ce pas paradoxalement cette suggestion qui au bout du compte nous reste des croisades ascétiquement méta-picturales menées par les artistes de Support/Surface?

R.-M. A. - La profondeur est dans la peau, dixit Valéry parce qu'elle nous met en communication avec l'immensité du monde extérieur. En même temps, elle est une surface d'échange avec l'intériorité. Or, puisque vous cherchez à traduire la complexité infinie de l'expérience sensorielle, où sont les points de contact de cette porosité ?

C. P. - Dans les déchirures constituantes qui produisent la «motilité », sans doute. Dans la mobilisation de tout ce qui, 
dans la langue, ne relève pas d'abord de la figuration ou de la nomination - et qui, du coup, peut-être, fixe des effets sensoriels, voire sensuels : l'écholalie, le découpage respiratoire des segments syntaxiques, la ponctuation rythmique, les effets métriques d'accélération ou de ralenti, la jouissance du babil glossolalique, une certaine façon d'immerger l'écrit dans une mastication syllabique régressive, l'idiotie assumée des gags formels, etc. En tout cas dans toutes les opérations dont on attend qu'elles ouvrent ce qui est en train de s'écrire sur des horizons dont celui qui écrit ne savait a priori rien et dont, in fine, il n'aura noté que la trace défective (c'est-à-dire ce qui, y important le souvenir du démesuré sensoriel, aura perturbé la mesure rationnelle de l'écrit).

R.-M. A. - L'écriture de Christian Prigent: à fleur d'émotion ou soigneusement distanciée?

C. P. - Credo (il synthétise tout ce que je viens de dire) : dans l'écriture, c'est la distance (un système de formalisation apathique) qui donne sa chance à une diction juste de l'émotion (l'excès ex-tatique).

R.-M. A. - Il y a un écart entre votre goût pour l'art brut et la sophistication de vos procédés. Ce qui n'empêche pas la lecture oralisée de vos textes d'en restituer la sauvagerie, oserai-je dire - mais telle une nature sinon domestiquée (heureusement) du moins canalisée par le verbe.

C. P. - «Sauvagerie », je ne dirais pas (j'ai un surmoi très très fort, vous savez; et un souci formel constant ; ma question est toujours : comment faire de l'art ?)... «Violence », si vous voulez... «Énergie », j'espère... Mes lectures publiques n'ont pas d'autre but que d'exposer les conditions dans lesquelles ça s'est 
écrit et de surligner par les moyens de l'oralité (rythmes, débits, sonorités, souffles $)^{9}$ certains des effets ambivalents que j'attends que produise l'écriture : les tensions contradictoires qui animent mes textes (comique et angoisse, violence et concertation rhétorique, trivialité et sophistication, intertexte érudit et babil enfantin, etc.). Je reviens là-dessus dans le volume Compile (un livre plus un CD) que P.O.L publiera début 2011. Ces lectures m'ont fait monter sur des scènes aux côtés de bien des poètes «sonores", des «performers", des " actionnistes», etc. Mais je ne suis pas vraiment proche de cela, qui ne m'intéresse pas très souvent (à quelques exceptions près, dont celle de Bernard Heidsieck, bien sûr). Entre autres parce que les artistes de ces courants sont souvent d'une insuffisance intellectuelle navrante. Et que leurs héritiers actuels me semblent rarement poursuivre un autre but artistique que la fixation d'un effet formel qui fait label — label qu'ils s'affairent alors à gérer, dans la logique marchande et spectaculaire de bien des aspects de l'art dit « contemporain ».

R.-M. A. - Et le «courant douloureux» qui pousse à écrire (Proust, p. 410)?

C. P. - Pas plus que le mot «écrivain » ne fonde une valeur (il désigne plutôt un souci), le mot « douleur » ne suffit évidemment à pourvoir d'authenticité et de profondeur une pratique artistique qui déclarerait $\mathrm{y}$ trouver sa source. Simplement, il m'a toujours semblé que mon besoin d'écrire (et de comprendre les causes et les effets de ce besoin) avait à voir avec une tentative de réponse à ces formes banales de la souffrance que sont l'angoisse, l'inertie dépressive, la mélancolie. À ce titre, cette souffrance m'apparaît donc

${ }^{9}$ Voir Prigent, 1984. 
effectivement fondatrice pour ce que j'ai été amené à écrire. Mais il n'y a à charger cela d'aucun pathos. Primo, parce que ce qui fait «courant» (ce qui porte à l'effort d'expression) ne relève d'abord ni de la psychologie ni de l'affect subjectif. La mélancolie dont je parle est celle de l'espèce. Le malaise, celui de la civilisation. L'inquiétude, celle du sens (de l'opacité du monde). L'intranquillité angoissée, celle de l'inadéquation des formes à la pression informe qui fait écrire. Deuzio, parce que le projet n'est pas d'exprimer ledit « courant douloureux » mais de détourner sa puissance de destruction pour défaire sous ses coups le décor aliénant du «monde » (les représentations qui cherchent à le pacifier en nous le légendant et en nous l'expliquant) ; et de répliquer à l'angoisse et à la dépression (qui sont des effets de la domination objectivée des représentations dont je parle) par la tonicité et l'inventivité de la forme écrite. Mes textes recherchent cet effet de tonicité entre autres choses par l'énergie phrasée, la vitalité bouffonne et le comique délibérément catastrophique du sens (je dis bien le comique pas l'humour, pas la finesse distinguée, pas le surplomb distancié, surtout pas « l'esprit »!).

R.-M. A. - «L'intranquillité intellectuelle, morale, sensuelle et sexuelle » (Prigent et Gorrillot, 2009, p. 20) dont vous parlez au sujet de la bibliothèque de votre père, en découle-t-elle seulement?

C. P. - Si les manifestations personnelles du « courant douloureux » propre à l'espèce et à sa culture s'avivent (voire naissent) de la fréquentation des livres; ou si ce sont elles qui nous poussent vers la bibliothèque - je n'en sais rien. Je sais seulement qu'aux environs de mes quinze ou seize ans, la banale «saison en enfer » de l'adolescence a coïncidé pour moi 
avec la lecture de Rimbaud. Je ne m'en suis jamais remis. Après, je suis allé lire de préférence ceux qui ont éprouvé (ou dit qu'ils éprouvaient) des choses semblables et pensé leur travail comme un mode de résolution de la crise à la fois subjective et... ontologique : Lucrèce, Hölderlin, Proust (chez qui j'ai trouvé la formule que vous citez), Artaud, Beckett... Ça avivait l'intranquillité, certes (en la figurant, en la nommant, en multipliant ses formes de manifestation symbolique). Mais ça proposait simultanément les exemples de triomphe sur elle que sont ces objets de jouissance esthétique et intellectuelle qu'on trouve dans les écrits des auteurs en question.

R.-M. A. - Sans tomber dans l'analyse facile, Lacan, quand même, on en parle?

C. P. - Lacan a été pour moi une référence théorique décisive. Explicite, souvent (voir mes entretiens avec le psychanalyste lacanien qu'est Hervé Castanet). Implicite plus souvent encore parce que je ne tiens jamais, n'ayant aucune compétence pour le faire, un discours de spécialiste (de psychanalyse lacanienne - ou de quoi que ce soit d'autre, d'ailleurs). Je ne me vois pas écrire « sur » Lacan. Je l'ai lu, pas in extenso, mais assez attentivement, dans les années 1970. Il m’en est resté quelques formules (sur la notion de "réel », sur les effets de vérité de l'acte signifiant manqué...). Parce qu'elles rencontraient et donnaient corps intellectuel à certaines de mes intuitions, ces formules ont été pour moi extraordinairement éclairantes, embrayeuses d'écriture et de pensée.

R.-M. A. - Et la fête linguistique de Rabelais ?

C. P. - Je n'ai pas vraiment lu Rabelais. Sinon au lycée, comme élève, puis comme professeur. Et plus tard, bien trop 
vite, pour introduire au numéro « La Dégelée Rabelais » de TXT, en $1987^{10}$. Ce que j'avais à en dire est dans les quelques pages que lui consacre mon essai Ceux qui merdRent. Elles ne vont pas au delà d'une mise en place élémentaire: Rabelais comme exemple d'une fiction à la fois bouffonne et savante, joueuse et combattante (portant le fer de la fiction dans la pensée rationnelle et sommant la fiction d'être à la hauteur des enjeux idéologiques d'époque). Rabelais, en somme, comme exemple et emblème de la littérature "carnavalesque " ${ }^{11}$ que voulait promouvoir dans les années 1970 l'avant-gardisme de TXT.

R.-M. A. - Umberto Eco vient de concevoir un programme d'expositions, de conférences et de concerts sur le thème de la liste (voir Eco, 2009). Or, contrairement à ce que l'on croit, celle-ci n'est pas toujours synonyme d'exhaustivité, de finitude, comme chez Rabelais, mais peut procéder de l'abîme, de la glose anxiogène, voire de la logorrhée. Vous, qu'est-ce qui vous intéresse dans ce type de dispositif ? Quelle y est la part d'épuisement, de soi et du réel ?

C. P. - Je ne vois pas une aussi grande fréquence de cette procédure dans mes livres. Elle m'a servi surtout au début des années 1980 pour composer quelques partitions destinées à l'exécution orale: les «Litanies de l'orgasme » ${ }^{12}$, la « Liste des langues que je parle», les «Pnigos »... C'était surtout pour disposer d'une forme simple, démonstrative, telle que la vitesse d'exécution propre à l'oral n'empêche pas qu'on en saisisse le dessin rythmique, qu'on en voie se former la sculpture acoustique. Et que dans la simplicité de cette forme les effets de

\footnotetext{
10 Voir TXT, n² 21, juin 1987, p. 3.

11 Voir Prigent, 1972a et Prigent et Pérez, 1981.

12 Écouter <http://www.le-terrier.net/musique/fleche.gif>.
} 
sens soient des effets d'épuisement respiratoire: une accumulation de langues inventées (comme une surenchère sur "l'infinité potentielle du code»!) ou un mime burlesque de l'obsession orgastique mise «à toutes les sauces » idiolectales. Pour le reste, s'il y a des listes ici et là dans mes livres de prose, elles sont variablement motivées. Il y a des recensements parodiques (la liste des possibles vocations "poétiques» du narrateur, dans Grand-mère Quéquette ${ }^{13}$ ) et des rafales de questions qui notent l'inquiétude vertigineuse de tous mes "personnages » face au chaos in-signifiant du monde. Mais je ne saurais cerner la raison unique, s'il y en a une, qui subsume ces variantes.

R.-M. A. - Je disais cela précisément pour le vertige, «l'expérience du précipice intérieur » de Ponge - le caillou qui peut se refermer —, mais aussi pour l'idée de précipitation et de précipité...

C. P. - C'est Georges Bataille qui s'inquiétait que la maladie logoscopique de Ponge ne le livre au vertige métaphysique qui peut s'ouvrir dans l'observation du moindre objet. À quoi Ponge, vous vous en souvenez, rétorquait fort pragmatiquement qu'il suffit de changer d'objet au dernier moment. C'était sa façon de geler le «souci» ontologique. Bon. Le "précipice intérieur» est là, toujours imminent, dans ce compagnonnage avec la psychose qu'on sait toujours à portée... d'expérience. Il se profile en particulier dans le sentiment de peu-de-réalité que nous donnent au jour le jour les images et les énoncés au travers desquels nous nous voyons sommés de nous représenter nos vies. Raison pour laquelle j'attends des actions d'écriture (quelques formes qu'elles prennent - les

13 Voir Prigent, 2003, p. 169-170. 
énumératives et les litaniques parmi d'autres) qu'elles soient davantage une réplique formelle (un précipité de densité pratique) à la béance psychotique qu'une plongée fascinée dans ses précipices.

R.-M. A. - La débauche lexicale, le carnaval sans fin du langage, n'est pas sans rapport avec la libido et les appétits ne sont pas négligeables dans votre œuvre ${ }^{14}$. D'où ma question : les mêmes énergies, sexuelle et poétique, sont-elles en jeu dans l'écriture?

C. P. — «Éros fait écrire » (c'est une formule de Ponge). Mais fait écrire essentiellement des banalités, des mièvreries ou des obscénités surindiquées. Et aucun poème d'amour ne s'élève à la valeur s'il n'est un tant soit peu « objectivé ». Ce qui veut dire en clair : s'il n'annule pas cyniquement son prétexte amoureux nominatif. Par exemple en le faisant disparaître derrière des contaminations formelles (Ronsard faisant jouer la généralité de l'anagramme aimer sous le nom de Marie) ou des perspectives cultivées (le même noyant sa Cassandre ou son Hélène dans le grand bain mythologique rempli par ces prénoms). C'est qu'il faut faire surgir la « chose » (l'objet in-fini, le réel) derrière le détail des corps individués et des objets ponctuels du désir. Pour que ça engage vraiment la puissance de défiguration des figures habituées du monde qu'est l'expérience d'Éros (ses exaltations, ses extases, ses abandons altruistes - mais aussi ses déroutes, ses hontes, ses violences, ses tractations gourmandes avec l'ignoble, le bestial et l'abject). Je voudrais quant à moi parvenir à élaborer des formes poétiques qui conservent en elles la trace de l'exaltation érotique et du pathos de fusion qui furent à leur origine. Mais

14 Voir Prigent, 1969b, 1981a, 1981b, 1999a, entre autres, et Collectif, 1981. 
qui livrent en même temps ce pathos et cette exaltation à la cruauté d'un jeu signifiant «carnavalesque». Un jeu activé par le savoir empirique que ça (la fusion, l'extase) n'a pas de lieu symbolique, ne se dit pas, ne peut que faire défaillir la représentation verbale. En somme: décomposer le lyrisme, sacrifier l'idéalisme effusif et renverser l'illusion du rapport (sexuel, verbal) réussi au profit d'un jeu verbal a-pathique, distancié et goguenard. En pariant que la vérité de l'expérience (la quantité d'inconnu qu'elle recèle) passera implicitement dans la tension que tente de maintenir une forme souveraine entre les deux registres opposés d'affects et de significations. C'est très précisément le chantier sur lequel je suis en ce moment (qui essaie de retraiter dans le sens que je viens de dire une masse de poèmes écrits à divers moments de ma vie sous l'effet de quelques effervescences érotico-amoureuses).

R.-M. A. - Quels rapports établissez-vous entre l'amour et la révolution?

C. P. - Voilà deux mots bien pesants. Peut-être faudrait-il éviter d'en user encore. Pourtant je vois bien qu'ils insistent. À peine me les glissez-vous qu'ils se mettent à clignoter de partout. Ces deux mots ont inscrit leur légende sous les images de ma vie comme sous celles de la vie de bien des gens. Parce que ce sont deux promesses : elles suggèrent qu'il existe des chances d'excéder la mortification mélancolique et l'abandon fataliste à "l'état des choses». Parfois même ces chances se réalisent (on aime, des barricades se lèvent, des murs tombent). Bien sûr, la «sagesse » du non-dupe revenu de tout que nous portons aussi en nous murmure que ce sont des leurres. Mais cette sagesse (outre que politiquement cynique) est stupide et mortifère. Si on écrit, c'est pour relever autant qu'on peut le défi 
que dessinent les deux promesses. Elles traversent en tout cas tout ce que j'ai essayé de penser, de ressentir, de styliser. Bien sûr, ça fatigue. Et ça se fatigue, ce double appel venu du fond des déserts du monde. Mais c'est intuable, nonobstant l'usure personnelle et les désespoirs politiques d'époque. Ce qu'il y a de commun entre les deux: l'utopie comme appel à excéder un présent politiquement injustifiable, éthiquement insupportable et subjectivement dépressif (quoiqu'on sache d'une part ce qui peut s'en suivre comme souffrances intimes et quoi qu'enseignent d'autre part les catastrophes sanglantes hélas historiquement avérées); le goût exalté du nouveau comme condition de la jouissance; une vocation à la perte (des certitudes, des repères) et à la destruction (des états stabilisés du lien social, de la pensée, du sentiment, du désir) comme prémisses d'une possible renaissance. Renaissance dont à sa façon le travail de régénération symbolique qui produit des livres étranges et un peu inquiétants est l'une des manifestations Cette manifestation est certes socialement discrète, peu partageable et effectivement fort peu partagée, sans guère d'effets contaminants vers l'extérieur - et en tout cas inapte à... révolutionner quoi que ce soit. Elle résiste, cependant. Et reste pour moi infiniment désirable. Parce que, sans sa prothèse de tonicité symbolique, rien du monde ne me paraît vraiment savoureux ni un tant soit peu intelligible.

R.-M. A. - Et la saveur, c'est important. D'où votre usage de l'ancien français dans Demain je meurs, par exemple?

C. P. - J'ai du goût pour la pluralité des langues, pour l'infinité du potentiel lexical, pour l'hétérogénéité des niveaux, des registres, des accents, pour les argots, les dialectes, les patois, les lexiques techniques. Il y a une saveur dans les 
prononciations et les phrasés variés, les énonciations bancales, les échos anciens toujours là, en sourdine, sous ce que le parler moderne en a fait. Travailler avec ça, c'est retraverser la mémoire de la langue. Et ce n'est pas sans émotion qu'on essaie de retrouver les façons que «ceux d'avant» avaient de la mastiquer et de la proférer. Je crois que l'effet que le monde nous fait et la vision que nous en avons s'enrichissent à proportion de la profusion des noms que nous posons sur lui. La justesse de ce que nous disons de lui est fonction de cette diversité polyphonique. Alors l'ancien français, oui - entre autres. Comme exemple d'une altérité historique de la langue dans la langue. Un moment qui m'émeut parce qu'inaugural, archaïque et séminal. Où j'aime aller chercher des formes syntaxiques, lexicales, métriques à la fois exotiques et familières (parce que la langue moderne ne les a pas oubliées : elles sont à l'œuvre dans les réseaux souterrains sur lesquels elle s'appuie).

R.-M. A. - Et si nous terminions par la fraîcheur que vous avez mentionnée au cours de nos échanges ? Quelle est-elle en fin de compte?

C. P. - Celle de cette vision dont je viens de parler. Écrire, c'est pour moi essayer de maintenir la fraîcheur de cette vision : sa singularité, sa résistance au lieu commun, son excès à l'idéologie, sa vocation à une réfection perpétuellement rejouée (contre l'inéluctable entropie, dans le temps, des significations organisées en discours et des figures composées en représentations). Pour continuer à penser dans l'ouvert, comme on disait jadis du côté de Tübingen. 


\section{Bibliographie}

BARTHES, Roland. 1972 (1953), Le Degré zéro de l'écriture suivi de Nouveaux Essais critiques, Paris, Seuil, coll. « Points ».

BATAILlE, Georges. 1930, «Espace », Documents, 2e année, $\mathrm{n}^{\circ} 1$, p. 41.

ColleCtif. 1981, Térature, n³ 3-4, «Le Sex o'clock de Christian Prigent », Rennes.

Eco, Umberto. 2009, Vertige de la liste, Paris, Flammarion (catalogue de l'exposition au Louvre, 2 novembre13 décembre 2009).

LEBEL, Jean-Jacques. 1965, Anthologie de la poésie de la Beat Generation, Paris, Denoël.

NAPOLI, Gabrielle. 2004, "Réel pour dire, réel à dire ", La Quinzaine littéraire, $\mathrm{n}^{\circ} 880,1^{\mathrm{er}}$ juillet, p. 10.

Ponge, Francis. 1965, Tome premier, Paris, Gallimard : Proêmes, « Des raisons d'écrire ».

Prigent, Christian. 1969a, «Pour Denis Roche», Action poétique, $\mathrm{n}^{\circ} 41-42$, p. 47-56;

—. 1969b, «Érotique voilée », Sud, n 1, p. 27-35;

—. 1971, « La Scène dans la Seine », TXT, n 3-4, p. 41-54;

—. 1972a, «Carnaval : inflation, réaction », TXT, n 5, p. 10-22;

—. 1972b, "Pour une poétique matérialiste », Critique, $\mathrm{n}^{\circ} 301$, p. $505-526$;

-. 1972c, "Ponge et le matérialisme», Cahiers du Centre international d'études poétiques, $\mathrm{n}^{\circ}$ 93, Bruxelles, p. 11-21;

—. 1974a, « Le Groin et le menhir », Critique, n³25, p. 529-540; 
-. 1974b, « Explication de texte », TXT, n 6-7, hiver, p. 61-81;

—. 1974c, « Deux notes », ibid., p. 103-111;

-. 1976, «La lessive du français d'église », Politique Hebdo, $\mathrm{n}^{\circ} 218,15-21$ avril, p. 28;

—. 1977a, Denis Roche, Paris, Seghers, «Poètes d'aujourd'hui » (essai);

-. 1977b, Power/powder, Paris, Christian Bourgois (fiction);

-. 1977c, "Le Texte et la mort», dans Philippe BonNefis et Pierre OSTER (dir.), Ponge inventeur et classique, Actes du colloque de Cerisy-la-Salle (2-12 août 1975), Paris, U.G.É., coll. « 10-18», p. 352-379;

—. 1978a, «Le Marquis de Sade n'ira pas au c.i.e.l. », Politique Hebdo, $\mathrm{n}^{\circ} 312$, 22-28 mai, p. 37-38;

-. 1978b, "La Besogne des mots chez Francis Ponge», Littérature, $\mathrm{n}^{\circ} 29$, p. 90-97;

—. 1980, «Liste des langues que je parle », repris dans PRIGENT et GORRILLOT, 2009, p. [240];

—. 1981a, Voilà les sexes, Paris, Luneau-Ascot (fiction);

-. 1981b, "Comment voir le sexe en peinture », Courrier $d u$ Centre international d'études poétiques, $\mathrm{n}^{\circ}$ 139-140, p. 25-29;

—. 1983, « Pnigos », Change, $n^{\circ} 42$, p. 90-94;

—. 1984, « La Voix de l'écrit », TXT, n 17, novembre, p. 33-38;

-. 1989a, Commencement, Paris, P.O.L (roman);

-. 1989b, « Le parti pris de Francis Ponge », TXT, n² 23, p. 54;

—. 1991, Ceux qui merdRent, Paris, P.O.L (essai);

—. 1993, « Wozu noch Dichter ?», TXT, n 31, juin, p. 74-77;

—. 1999a, Le Professeur, Paris, Al Dante; 
—. 1999b, «Un peu de petite histoire », Action poétique, $\mathrm{n}^{\circ} 153-$ 154, janvier, p. 48-49;

—. 2003, Grand-mère Quéquette, Paris, P.O.L (fiction);

-. 2005, [sans titre,] dans Écrire, pourquoi?, Paris, Argol, p. 141-143;

—. 2007, Demain je meurs, Paris, P.O.L (roman);

—. 2010, Météo des plages, Paris, P.O.L (roman en vers).

- et Hervé CASTANET. 2004, Ne me faites pas dire ce que je n'écris pas, Saussines, Cadex (entretiens);

- et Bénédicte Gorrillot. 2009, Christian Prigent, quatre temps. Rencontre avec Bénédicte Gorrillot, Paris, Argol, coll. « Les Singuliers » (entretiens);

- et Mathias PÉREZ. 1981, 200 Conseils pour un carnaval, Rennes, Térature (ouvrage illustré).

Proust, Marcel. 1989, À la recherche du temps perdu, édition de Jean-Yves TADIÉ, Paris, Gallimard, coll. «Bibliothèque de la Pléiade ", t. IV : Le Temps retrouvé.

Roche, Denis. 1972, Le Mécrit, Paris, Seuil.

-. 1977, "Les poudres et les pouvoirs de Christian Prigent», Le Monde des livres, $1^{\mathrm{er}}$ juillet, p. 19.

- et Roger-Michel ALLEMAND. 2001, «L'Aléa littéraire. Entretien sur l'écriture et la photographie », Ariane, n 17, dossier «Le Cercle des muses », Lisbonne, Groupe Universitaire d'Études de Littérature Française, p. 273-302. 